\title{
NON-TECHNICAL LOSS DIAGNOSIS IN ELECTRICAL NETWORKS WITH A RADIAL LAYOUT
}

\author{
AnNa I. PÓzNa *1, ATtILA Fodor ${ }^{1}$, ANd KATALIN M. HANGos ${ }^{2}$ \\ ${ }^{1}$ Department of Electrical Engineering and Information Systems, University of Pannonia, P. O. Box 158, \\ Veszprém, 8201, HUNGARY \\ ${ }^{2}$ Institute for Computer Science and Control, Hungarian Academy of Sciences, P. O. Box 63, Budapest, \\ 1518, HUNGARY
}

\begin{abstract}
A network-oriented non-technical loss detection and localization methodology is presented in this paper. The basic idea behind the proposed methodology is the deviation of the measured voltages from their nominal values. The operation of the algorithm was investigated by simulation experiments using an (IEEE) European Low Voltage Test Feeder benchmark network. The simulation results show that the proposed method is able to detect and localize multiple occurrences of nontechnical losses caused by fraudulent meters.
\end{abstract}

Keywords: power network, diagnosis, non-technical loss, load-flow

\section{Introduction}

The demand for electrical energy is continuously increasing on a global scale. Electrical energy is generated by power plants or renewable energy sources and is transmitted from the source of generation to consumers through distribution stations and power lines. During transmission, technical losses, that originate from dissipation in conductors, transmission lines and substation transformers as well as magnetic losses in transformers, reduce the efficiency of power delivery. The proportion of technical losses is about $20 \%$ of the total energy transmitted.

Besides the technical losses, non-technical losses (NTLs) may be present as well. These are unnecessary losses which are not expected and cannot be anticipated. The NTLs are usually related to energy theft and fraudulent consumption behavior. Energy theft has been a widespread and major issue for many years and various techniques of energy theft are present from unregistered users to hacking meters [1]. Following this unwanted phenomena, several methods of non-technical loss detection have appeared in the literature. It can be stated that no golden rule exists for detecting energy theft, rather, several different approaches are available [2]. Papers [3] and [4] provide very good reviews on the most frequently used methods in this field.

The majority of solutions available in the literature are based on the analysis of consumption data using some statistical or machine-learning methods, for example, the authors of [5] used a linear regression-based pro-

\footnotetext{
*Correspondence: pozna.anna@virt.uni-pannon.hu
}

cedure that not only detects energy theft but defects of smart meters as well. A probabilistic neural networkbased classification approach is presented in [6] where the Levenberg-Marquardt method is used for training the network. A support-vector machine-based solution is given in [7], where a parallel computer architecture was proposed in order to enhance computation.

Another approach to non-technical loss detection is based on network topology, such network-oriented methods use readings from grid sensors and smart meters. In [8], the authors proposed state estimation with a Kalman filter to identify currents and biases in a microgrid network. The currents and biases are estimated separately using two different filters. If the estimated bias of a customer exceeds the predefined threshold, then this user has committed fraud. The authors of [9] suggest a probabilistic power flow approach to NTL detection. The output of the algorithm is a probability distribution of NTLs in the subnetwork.

Besides the above classes, other methods of localizing illegal electricity usage exist, e.g. in [10] a power lines inspection robot was applied to find NTLs.

The approach followed in the present work belongs to the network-oriented class and it is based on analyzing the differences between the measured and nominal voltages. The uncertainty in the model parameters together with the measurement uncertainties are taken into account to ensure the approach is applicable to real-world cases.

The structure of the paper is as follows. The basic notions and problems are introduced in Section 2. Section 3 
contains the main contribution of the paper and presents the proposed novel diagnostic method in detail. Afterwards, the proposed method is subject to simulationbased analysis in Section 4.

\section{Problem statement}

\subsection{Non-technical losses}

According to a source Non-Technical Losses (NTLs) can be classified as follows [11]:

Before meter: illegal tapping of distribution lines or feeders

Meter: inaccurate power readings due to a meter being faulty (e.g. changes to the calibration), reversed, disconnected or bypassed

Billing: non-payment of electricity bills, inaccurate billing, faulty operation of the billing system, a cyber attack against the billing system, etc.

NTLs caused by companies in the US were estimated to cost between $0.5 \%$ and $3.5 \%$ of annual gross revenues. The NTLs may account for more than $15 \%$ of the generated power in some countries [2] and [12].

In the remaining part of the paper only the following type of non-technical loss is examined that belongs to the first class above:

- fraud committed by tampering with the meter (reversing or hacking the hardware or software, or calibration of the meter),

- bypassing the meter.

\subsection{Detection and localization}

In general, two main parts of the diagnostic procedure exist: fault detection and fault identification. The aim of detection is to decide if any fault has occurred in the system. The exact type and localization of the occurred fault is determined in the identification phase. In the case of non-technical loss diagnosis, detection and localization are defined as follows:

Detection of non-technical loss means that the loss is acknowledged.

Localization means that the fraudulent user is identified if the illegal consumption of power occurs at the meter. In the case of multiple NTLs, every fraudulent meter is to be identified by the method.

Illegal load is a load that originates from a fraudulent meter, i.e. the consumer uses illegal power.

\subsection{Basic assumptions}

During development of the non-technical loss detection and localization method, the following assumptions were made:

- The electrical network is represented by its static linear model. The known parameters of the model are the resistances of the transmission lines, the current and voltage of the feeding point (transformer), and the currents of the loads.

- The structure of the electrical network is radial (for more details see Section 3.2).

- Every load has a smart meter that measures the current, voltage and power consumption of the load.

- At least one illegal load may be present in the network. If more than one illegal load exists then each is located in a different part of the network (see Section 3.2 for more details).

\subsection{Uncertainties and measurement errors}

The main uncertainties that affect the voltages and currents in a public electrical grid can be classified as follows:

- Uncertainties about the parameters of the transmission lines

The resistance of the transmission lines is the main source of uncertainty. This resistance can be computed from its diameter, curvature and length, which are functions of temperature. The function is approximately linear in the domain $-50{ }^{\circ} \mathrm{C}$ to $100{ }^{\circ} \mathrm{C}$ :

$$
\rho=\rho_{0}\left(1+\alpha\left(T-T_{0}\right)\right),
$$

where $\rho$ and $T$ stand for the values of resistance and temperature, respectively, while $\rho_{0}$ and $T_{0}$ denote the nominal resistance and temperature, respectively, and finally $\alpha$ represents the temperature modulus. The main uncertainty over the line is the small difference between the planned and installed transmission line. The losses, which are justifiable given these technical reasons, contribute to approximately $2-3 \%$.

\section{- Measurement errors}

The presence of smart meters in our electrical network is assumed. The precision of smart meters varies from $\pm 0.2 \%$ to $\pm 2 \%$ depending on their precision and the percentage of nominal power, see International Standards IEC 62051, IEC 62052-11, IEC 62052-21 and IEC 62052-31.

In this paper the effects of the harmonic currents [13] are not investigated, rather, it is assumed that the voltages and currents are sinusoidal. The effects of asymmetrical loads [14] are also not investigated because a single-phase grid is assumed. A three-phase grid can be assembled from three single-phase grids with one $N$ line. 


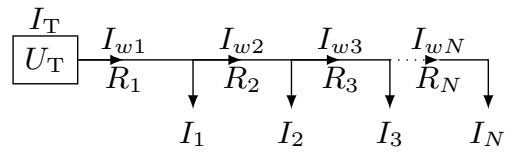

Figure 1: Single-feeder layout

\section{Diagnostic method}

The proposed diagnostic method uses the topology and electrical parameters of the network together with the nominal and measured voltages as well as current values to detect and localize the illegal loads. The nominal voltage and current values are generated from the topology and the parameters of the network determined by solving its linear time-invariant model using the known feeder voltage and current values [15].

Input data It is assumed that the model of the network is present together with the nominal values of its elements. Current and voltage readings with measurement errors are available from smart meters of all loads.

Detection The proposed diagnostic method is based on analyzing the difference between the measured and nominal voltages. It is assumed that the currents measured are the nominal currents of the network. The presence of an illegal load is detected if a difference between the sum of the measured currents $\left(\tilde{I}_{i}, i=1, \ldots, N\right)$ and the measured current of the transformer $\left(I_{\mathrm{T}}\right)$ exists.

$$
\sum_{i=1}^{N} \tilde{I}_{i}-I_{\mathrm{T}}>\varepsilon
$$

Localization The localization method starts with the simulation of the network assuming the nominal current values. During the simulation, the voltages of the loads are computed. The simulated voltages are considered to be the nominal voltages. Subsequently the nominal voltages are compared to the measured voltages. Localization is based on the evaluation of deviations in voltage from the nominal values. The measurement error is taken into account in such a way that only deviations that fall outside the maximum measurement error are considered during the diagnosis.

\subsection{Single-feeder layout}

The single-feeder layout is the simplest topology of electrical networks. It contains a single feeding point with several loads connected to it along a transmission line (Fig. 1). The difference between the nominal and measured voltage levels is computed as:

$$
\Delta U_{i}=\tilde{U}_{i}-U_{i}, i=1, \ldots, N
$$

where $\tilde{U}_{i}$ and $U_{i}$ are the measured and nominal voltages of the $i$ th load, respectively. Larger drops in voltage than the nominal ones are caused by illegal loads. Therefore,

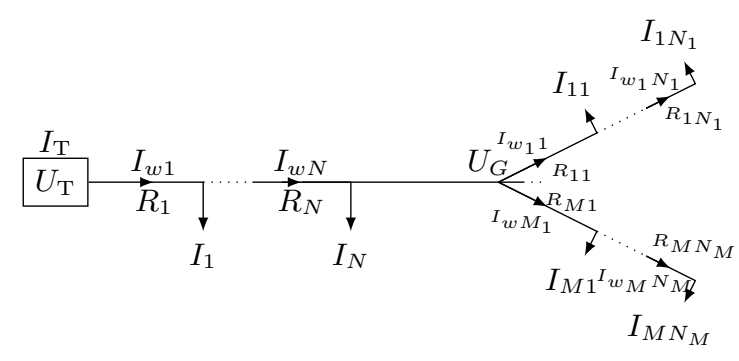

Figure 2: Radial feeder layout

the value of $\Delta U_{i}$ is negative. If the illegal load is in the $k$ th load, differences in voltage of subsequent loads are equal to the $k$ th load. This difference is proportional to the magnitude of the illegal current and the resistance of the transmission line:

$$
\Delta U_{i}= \begin{cases}I_{\mathrm{ill}} \sum_{j=1}^{i} R_{j}, & \text { if } i<k \\ I_{\mathrm{ill}} \sum_{j=1}^{k} R_{j}, & \text { if } i \geq k\end{cases}
$$

If, by starting from a certain load, the voltage differences of the loads are equal, then the load in question consumes power illegally. In practice two differences in voltage are considered to be equal if the difference between them is less than the measurement error. Therefore, localization of the illegal load is quite straightforward in a singlefeeder layout: the load in question needs to be identified and from this the differences in voltage start to become equal.

This method can be generalised if more than one illegal load in a single-feeder network is present. In this case, sections in the sequence of voltage differences where consecutive voltage differences are equal exist. The illegal loads are located at the start of these sections.

\subsection{Radial layout}

The radial-feeder layout is commonly used in lowvoltage networks. The general structure of a radial-feeder network can be seen in Fig. 2. This type of network can be decomposed into single-feeder subnetworks by identifying and cutting off the branches (for further details see [16]).

The loads are considered to be part of the same subnetwork if they are connected to the same bus. After decomposition, a set of disjoint single-feeder networks is formed which can be processed in parallel.

From a diagnostic point of view, two types of subnetworks should be distinguished. The first type is when the subnetwork contains more than one load (referred to as multiple load subnetwork hereinafter). The structure of this subnetwork is similar to the single-feeder layout (Fig. 1 ). The second type of subnetworks is the special case when the subnetwork contains only one load (referred to as single load subnetwork hereinafter).

One illegal load in a multiple load subnetwork If only one illegal load is present in the whole network and it is 
located in a subnetwork with several loads, then it can be clearly localized using the diagnostic method described in Section 3.1.

One illegal load in a single load subnetwork In the case when the subnetwork contains only one load, the voltage difference cannot be compared to any other voltage difference within this subnetwork. Therefore, the diagnostic procedure in Section 3.1 cannot be used. In this case, the voltage deviations need to be analyzed globally. The increased consumption causes the biggest deviation in the voltage at the location of the illegal load. In this case, the diagnostic procedure to determine the minimum voltage difference is identical.

More illegal loads in multiple load subnetworks If more than one illegal load is present and are located in different multiple load subnetworks, then their locations can be determined independently of each other.

More illegal loads in single load subnetworks In this case, local minima in the voltage differences indicate the locations of the illegal loads. The local minima are determined in such a way that the voltage differences of the single loads can be compared to the voltage differences of their two nearest neighbors. If the voltage difference of the single load is smaller than that of its neighbors, then an illegal load is present at the single load.

\subsection{Diagnostic algorithm}

The four aforementioned cases in the radial layout can be merged into one algorithm, the flowchart of which can be seen in Fig. 3. During the diagnosis the illegal loads identified are collected in a set called NTL. At the beginning of the diagnostic algorithm, the set $N T L$ is empty. The diagnostic algorithm searches for illegal loads in different parts of the network. The algorithm consists of three main parts: searching in multiple load subnetworks, searching for one illegal load in single load subnetworks, and searching for more illegal loads in single load subnetworks.

\section{Detection}

The inputs of the combined algorithm are the measured currents and voltages as well as the network structure. First, to detect the illegal consumption, the inequality in Eq. 2 is checked. If the difference between the sum of the measured currents and the transformer current exceeds a predefined threshold, then illegal consumption occurs in the network, otherwise the network operates normally. If illegal loads are detected, then the algorithm tries to determine their locations. To do this, the network is simulated using the measured current to obtain the nominal voltage values.

\section{Isolation}

NTLs in multiple load subnetworks This algorithm starts to search for illegal loads in the multiple load subnetworks using the method described in Section 3.1. The illegal loads identified are added to the set $N T L$. In this step, all of the illegal loads in the multiple load subnetworks are localized.

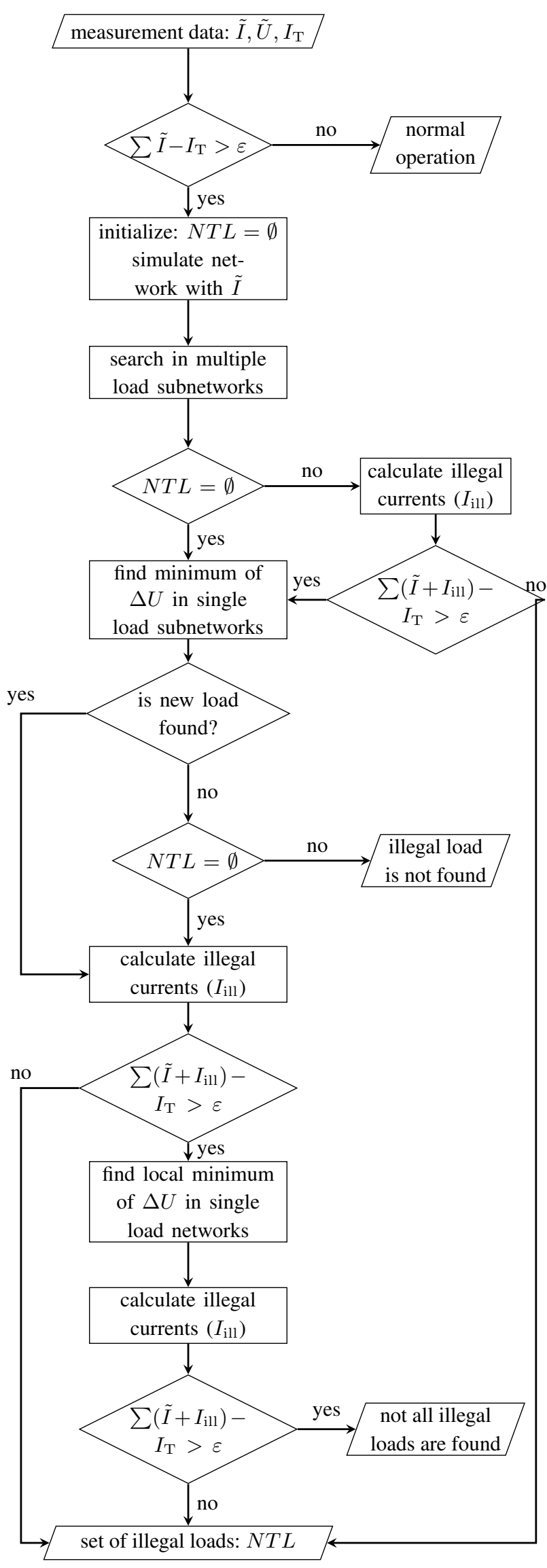

Figure 3: Flowchart of the diagnostic procedure 


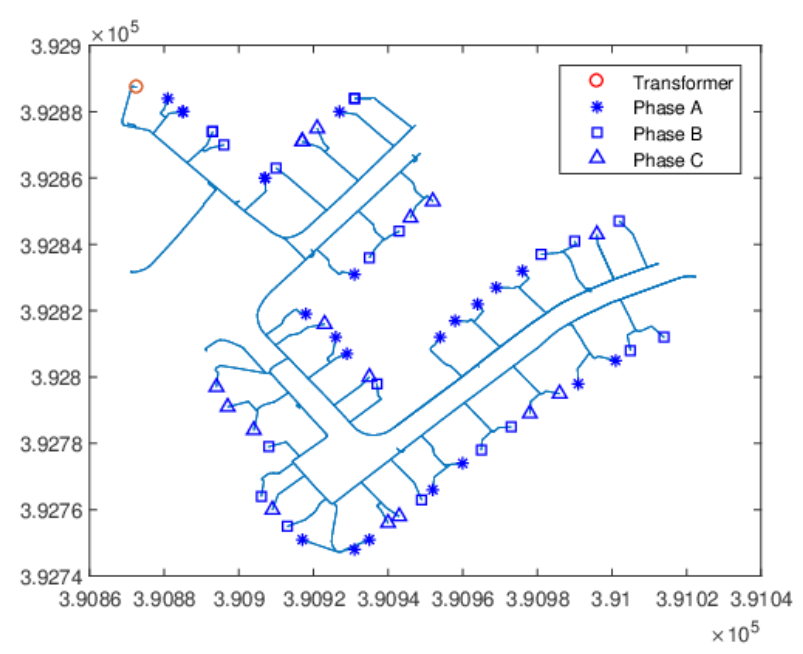

Figure 4: Structure of the IEEE 2015 European Low Voltage Test Feeder network

Before proceeding, the algorithm checks if any illegal loads have been identified in the multiple load subnetworks. If the set NTL is empty, then no illegal loads were found, therefore, they should be located in the single load subnetworks. If at least one illegal load is identified in the multiple load subnetworks, then their illegal currents are calculated using Eq. 4. The currents of the illegal loads are substituted by the calculated currents and the inequality of Eq. 2 is checked. If the inequality is false, then all of the illegal loads are localized and the algorithm stops. If the inequality is true, then at least one illegal load is still present in the single load subnetworks.

NTL in a single load subnetwork To identify the illegal load in single load subnetworks, the algorithm searches for the minima of the voltage differences. The illegal load possesses the minimum voltage difference.

If during this step no new illegal loads are identified, then two scenarios are possible: ( $i$ ) if set NTL is still empty, then the illegal load has not been found and the algorithm proceeds to the second search in the single load networks. (ii) If the set NTL is not empty, then the illegal currents are calculated and the inequality of currents checked. If no significant difference is present, then all illegal loads have been identified and the algorithm stops. If the inequality is true, then a second search of the single load networks is performed.

More NTLs in single load subnetworks During this step, the voltage difference of the single loads is compared to the voltage difference of their two nearest (left and right) neighbors. If the voltage difference of the single load is minimal between the three differences, then the single load is an illegal load.

After this search the inequality of the currents is verified again. If a difference is still present, then not all the illegal loads have been identified, otherwise all of the illegal loads have been found and the algorithm stops.

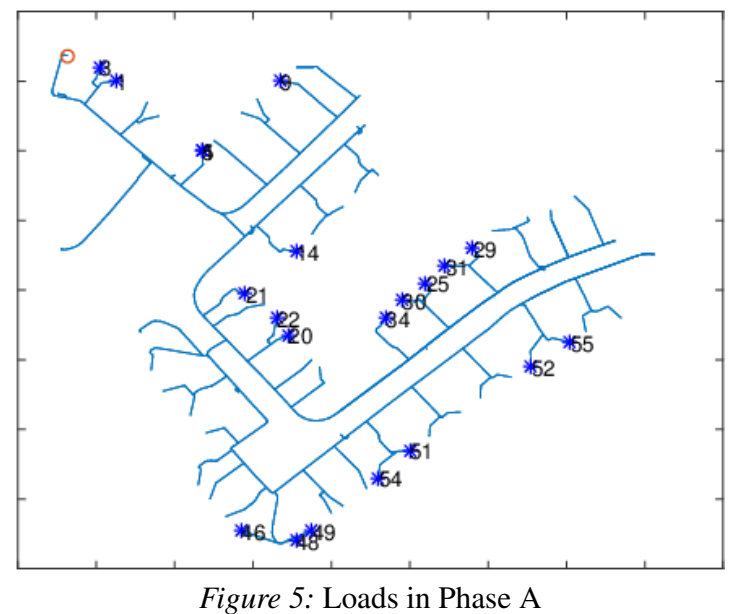

\section{Case study}

The diagnostic algorithm was tested on the IEEE 2015 European Low Voltage Test Feeder [17] which is a benchmark provided by the Power System Analysis, Computing \& Economics (PSACE) Committee. It is a threephase radial distribution feeder with one feeding point. The network contains 1 transformer, 55 loads and 905 lines. The structure of the network can be seen in Fig. 4 .

The algorithm was tested by only taking into consideration one phase (namely Phase A) of the system. 21 loads are present in this phase which are displayed in Fig. 5 along with their identifiers. At the time of the test, the minimum and maximum power consumed by these loads was $35 \mathrm{~W}$ and $64.9 \mathrm{~W}$, respectively. During the diagnosis, the measurement error was set at $0.2 \%$ of the measured currents. The network belonging to Phase A can be decomposed into 13 subnetworks, 5 of which are single load networks and 8 are multiple load networks consisting of 2 loads each.

The decomposition and diagnostic algorithm was implemented in MATLAB. The simulation of the network was also performed in MATLAB [18] using the method of nodal potentials.

\subsection{Case 1: One illegal load}

At first, it is assumed that only one illegal load is present in the network, more specifically in a single or multiple load subnetwork. Let us consider the subnetwork that contains the loads No. 25 and 30. Load No. 25 increases its consumption by $80 \%$ of its nominal value but deceives the current meter, therefore, the registered current value is not suspicious. The network is simulated using the nominal current values.

The difference between the nominal and measured voltages is presented in Fig. 6. It can be seen that the voltage differences are equal in the multiple load subnetworks, e.g. loads 1 and 3 as well as 20 and 22, except for the subnetwork of loads 25 and 30 . Since the absolute voltage deviation at load No. 25 exceeds that at load No. 30 , the illegal consumption is located at load No. 25 . 


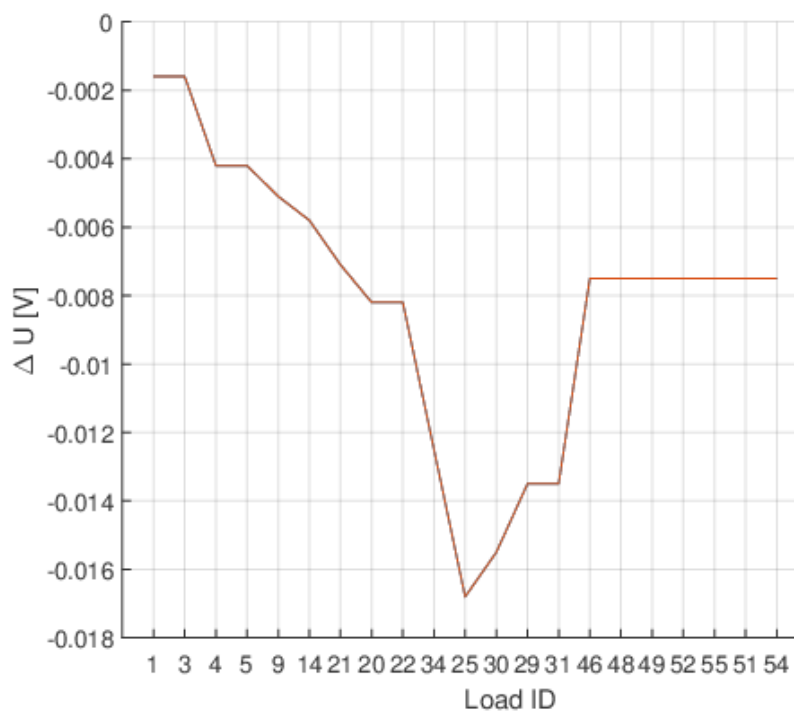

Figure 6: Voltage differences of the loads in Case 1

\subsection{Case 2: More illegal loads in different sub- networks}

In the second case, it is assumed that two illegal loads are present in different subnetworks. The first illegal load is No. 46 which is located in a single load subnetwork. The power consumption of load No. 46 is $50 \%$ more than its nominal value. The second illegal load is load No. 54 which is located in a multiple load subnetwork along with load No. 51. The consumption of load No. 54 is increased by $80 \%$ of its nominal value.

The difference between the simulated and measured voltages can be seen in Fig. 7. At first the diagnostic algorithm searches for illegal loads in the multiple load subnetworks. In the subnetwork of loads No. 51 and 54, the voltage deviation of load No. 54 exceeds the voltage deviation of load No. 51, therefore, load No. 54 is identified as an illegal load. The illegal current is calculated using Eq. 4. In the other multiple load subnetworks, the voltage differences of the loads within a subnetwork are equal, therefore, it can be stated that no illegal loads are present in these subnetworks.

The algorithm checks if some remaining illegal currents are present thereafter. A difference between the current of the transformer and the sum of the measured currents is still present which is indicative of at least one illegal load that is yet to be identified. These illegal loads should be located in single load networks. The minimum of the voltage differences is located at load No. 46, therefore, it is an illegal load. After calculating the illegal current of load No. 46 and checking the inequality in Eq. 2, it can be stated that no additional illegal loads are present in the network.

\section{Conclusions and future work}

A novel diagnostic method for detecting and locating illegal loads in electrical radial networks is proposed in this

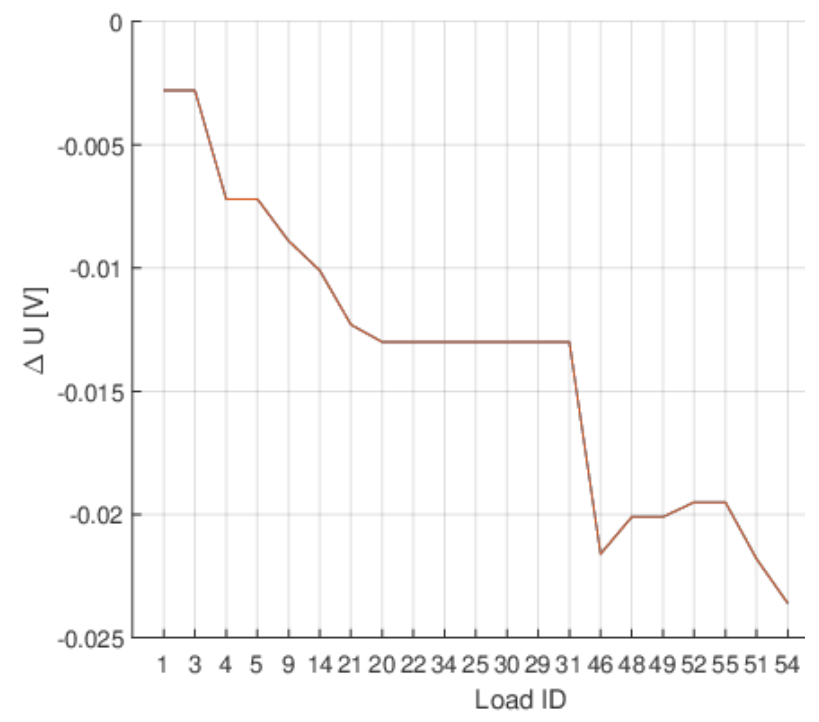

Figure 7: Voltage differences between the loads in Case 2

paper that utilizes the topology of the network and is capable of taking the uncertainties and measurement errors into account.

A preprocessing step decomposes the radial layout of single-feeder subnetworks with single or multiple loads, and the method is capable of locating the illegal load(s) in the subnetworks in parallel. The proposed method can detect and locate multiple independent illegal loads under certain conditions.

Future work will include the extension of the diagnostic methods to general, not necessarily radial, topology and to develop the computational model of a network to handle the uncertainties related to network parameters (resistances).

Furthermore, the effect of the uncertainties and measurement errors on the diagnostic accuracy should also be investigated.

\section{Acknowledgement}

We acknowledge the financial support of Széchenyi 2020 under the EFOP-3.6.1-16-2016-00015. We acknowledge the financial support of Széchenyi 2020 under the GINOP-2.2.1-15-2017-00038. This research is partially supported by the National Research, Development and Innovation Office - NKFIH through grant No. 115694.

\section{REFERENCES}

[1] Ahmad, T.; Chen, H.; Wang, J.; Guo, Y.: Review of various modeling techniques for the detection of electricity theft in smart grid environment, $R e$ new. Sust. Energ. Rev., 2018 82, 2916 - 2933, DOI: 10.1016/j.rser.2017.10.040 
[2] Smith, T.B.: Electricity theft: a comparative analysis, Energy Policy, 2004 32(18), 2067 - 2076, DOI: 10.1016/S0301-4215(03)00182-4

[3] Messinis, G.M.; Hatziargyriou, N.D.: Review of non-technical loss detection methods, Electr. Pow. Syst. Res., 2018 158, 250 - 266, DOI: 10.1016/j.epsr.2018.01.005

[4] Viegas, J.L.; Esteves, P.R.; Melício, R.; Mendes, V.; Vieira, S.M.: Solutions for detection of nontechnical losses in the electricity grid: A review, $R e$ new. Sust. Ener. Rev., 2017 80, 1256 - 1268, DOI: 10.1016/j.rser.2017.05.193

[5] Yip, S.C.; Wong, K.; Hew, W.P.; Gan, M.T.; Phan, R.C.W.; Tan, S.W.: Detection of energy theft and defective smart meters in smart grids using linear regression, Int. J. Elec. Power, 2017 91, 230 - 240, DOI: 10.1016/j.ijepes.2017.04.005

[6] Ghasemi, A.A.; Gitizadeh, M.: Detection of illegal consumers using pattern classification approach combined with Levenberg-Marquardt method in smart grid, Int. J. Elec. Power, 2018 99, 363 - 375, DOI: 10.1016/j.ijepes.2018.01.036

[7] Depuru, S.S.S.R.; Wang, L.; Devabhaktuni, V.; Green, R.C.: High performance computing for detection of electricity theft, Int. J. Elec. Power, 2013 47, 21 - 30, DOI: 10.1016/j.ijepes.2012.10.031

[8] Salinas, S.A.; Li, P.: Privacy-preserving energy theft detection in microgrids: A state estimation approach, IEEE T. Power Syst., 2016 31(2), 883-894, DOI: 10.1109/TPWRS.2015.2406311

[9] Neto, E.A.A.; Coelho, J.: Probabilistic methodology for Technical and Non-Technical Losses estimation in distribution system, Electr. Pow. Syst. Res., 2013 97, 93-99, DOI: 10.1016/j.epsr.2012.12.008

[10] Oyun-Erdene, M.; Byambasuren, B.E.; Matson, E.T.; Kim, D.: Detection and localization of illegal electricity usage in power distribution line, $M u l$ timed. Tools Appl., 2016 75(9), 4997-5012, DOI: 10.1007/s11042-014-2022-2
[11] Ahmad, T.: Non-technical loss analysis and prevention using smart meters, Renew. Sust. Energ. Rev., 2017 72, 573-589, DOI: 10.1016/j.rser.2017.01.100

[12] Nagi, J.; Mohammad, A.; Yap, K.; Tiong, S.; Ahmed, S.: Non-technical loss analysis for detection of electricity theft using support vector machines, in Power and Energy Conference, 2008. PECon 2008. IEEE 2nd International (IEEE), 907912, DOI: 10.1109/PECON.2008.4762604

[13] Görbe, P.; Magyar, A.; Hangos, K.M.: THD reduction with grid synchronized inverter's power injection of renewable sources, in Power Electronics Electrical Drives Automation and Motion (SPEEDAM), 2010 International Symposium on (IEEE), 1381-1386, DOI: 10.1109/SPEEDAM.2010.5545079

[14] Neukirchner, L.; Görbe, P.; Magyar, A.: Voltage unbalance reduction in the domestic distribution area using asymmetric inverters, J. Clean. Prod., 2017 142, 1710-1720, DOI: 10.1016/j.jclepro.2016.11.119

[15] Greber, M.: Non-technical loss detection in electrical distribution network (in Hungarian), Tech. Rep., Faculty of Information Technology, University of Pannonia, 2018

[16] Pózna, A.; Fodor, A.; Gerzson, M.; Hangos, K.: Colored Petri net model of electrical networks for diagnostic purposes, IFAC-PapersOnLine, 2018 51(2), 260-265, DOI: 10.1016/j.ifacol.2018.03.045

[17] Schneider, K.; Mather, B.; Pal, B.C.; Ten, C.W.; Shirek, G.; Zhu, H.; Fuller, J.; Pereira, J.L.R.; Ochoa, L.; De Araujo, L.; et al.: Analytic Considerations and Design Basis for the IEEE Distribution Test Feeders, IEEE T. Power Syst., 2018 33(3), 3181-3188, DOI: 10.1109/TPWRS.2017.2760011

[18] The Mathworks, Inc., Natick, Massachusetts: MATLAB version 9.0.0.341360 (R2016a), 2016 